\title{
A NOMA-Combined Hybrid Approach for SDMA Improvement in Wireless Powered Communication Networks
}

\author{
Juhyun Maeng ${ }^{1} \cdot$ Mwamba Kasongo Dahouda $^{1} \cdot$ Inwhee Joe $^{1}$ (D)
}

Accepted: 8 August 2021 / Published online: 25 August 2021

(c) The Author(s) 2021

\begin{abstract}
Wireless Powered Communication Network (WPCN) consists of Hybrid Access Point (HAP) that performs power transmission and data collection at the same time, and multiple nodes that can transmit data. In WPCN, depending on the wireless communication environment, the nodes cannot be able to transmit data because they can fail to receive power. Hence, increasing the transmission rate under a given resource is one of the very important issues. In ordinary mobile communications, a cell is divided into several sectors and the data is collected through multiple antennas to increase the transmission rate using SDMA. As a result, if the number of nodes in the one sector increases, the interference between nodes increases, and the transmission rate may decrease. Accordingly, in order to maximize performance, the number of nodes that can exist in a sector must be limited. The transmission rate between nodes according to the distance difference may not be fair because the nodes far from the HAP charge a small amount of power by attenuation of the signal, and the nodes close to the HAP charge a relatively large amount of power. Therefore, we propose Hybrid SDMA and Non-Orthogonal Multiple Access (NOMA) as a way to maximize the performance in term of both Sum-Throughput and Fairness. Also, we prove that there is a tradeoff between Sum-Throughput and Fairness according to the number of sectors. The simulation results show that the Hybrid SDMA and NOMA improves the performance substantially compared to the conventional SDMA.
\end{abstract}

Keywords WPCN $\cdot$ MISO $\cdot$ SDMA $\cdot$ NOMA

Inwhee Joe

iwjoe@hanyang.ac.kr

Juhyun Maeng

jhmaeng@hanyang.ac.kr

Mwamba Kasongo Dahouda

dahouda37@hanyang.ac.kr

1 Computer Software Department, Hanyang University, 222 Wangsimni-ro, Seongdong-gu, Seoul 04763, South Korea 


\section{Introduction}

In wireless sensor networks, power management of battery-powered nodes is critical; insufficient power on a particular node can cause network instability due to a node that is not operating. Network instability can cause the collection of sensitive data to fail because communication between nodes is not performed. In addition, the nature of nodes that require battery replacement periodically when deploying nodes for configuring wireless sensor networks is constrained to retire nodes while considering accessibility to them.

In order to overcome this limitation, a technology that can more efficiently manage the battery of a node is Wireless Powered Communication (WPC). This technology is divided into Simultaneous Wireless Information and Power Transfer (SWIPT) and Wireless Powered Communication Networks (WPCN), and it consists of Hybrid Access Point (HAP) and Wireless Devices (WD). SWIPT simultaneously transmits power and data from the HAP to the WD over the same Radio Frequency (RF) signal in the downlink (DL). On the other hand, WPCN transmits power from HAP to WD with other RF signals; data is sent from WD to HAP via uplink (UL). The WD receives power through SWIPT; therefore, only the power harvested by WD is needed, and the power collected (Energy Harvesting) by the WD must be used for data transfer and Information decoding (ID). Therefore, it is essential to improve the uplink transmission rates for WD in WPCN [1].

Recently, research on systems that collect data using nodes and provide various functions with collected data has been active. WPCN is a suitable technology for such systems; it can contribute to the development of systems that provide various functions with collected data if the limitations of WPCN can be improved. The WPCN consists of a HAP that transmits power wirelessly to the WD to support the charging of WD's batteries, and the WD transmits data to the HAP using the received power. Through this WPCN, the WD's batteries can be regularly charged to ensure the stability of the network composed of multiple WD's batteries, and the WD's batteries can be charged wirelessly to solve the difficulties of WD deployment. However, although WPCN can increase the efficiency of WD's battery management, and it seems that it cannot guarantee the operation of WD; this is by reason of the transmission of power to the WD is broken due to radio wave failure, and there are often situations in which the power required for WD operating cannot be reliably supplemented. Therefore, it is necessary to improve the transmission rate with the power that the WD is collecting up to prevent data transmission failures due to lack of power. Reducing data loss while minimizing the power reduction of propagation will be one of the ways to speed up the transmission rate. In conventional mobile communication, one cell is divided into multiple sectors to reduce interference between nodes while reducing the power loss of each node. Also, the transmission rate is improved by receiving data transmitted by each sector per node through multiple base stations.

In this paper, one of the multiple antenna technologies, Multiple-Input Single Output (MISO), and Multiple antenna technologies such as Space Division Multiple Access (SDMA) and Non-Orthogonal Multiple Access (NOMA), which are candidates for 5G mobile communication technology, will be used to improve transmission rates. MISO can increase the amount of data that HAP can receive over multiple antennas and reduce the power consumption of battery-powered nodes with a single antenna. Furthermore, SDMA can reduce interference between sectors through sector division, and NOMA can reduce interference between WDs within the sector. As a base station, HAP periodically transmits power to all WDs in the cell via radio waves. Each WD that receives this power simultaneously transmits data collected by different signal strength to HAP depending on the 
distance difference from the base station. For example, a WD located far from the HAP transmits data with a strong signal strength, whereas a nearby WD transmits data with weak signal strength. Signals from WDs are superimposed by sector and sent to HAP. HAP receives overlapping signals from each sector by antenna simultaneously via Hybrid SDMA and NOMA Protocol, and NOMA-based overlapping signals from each sector collected by antenna via SDMA are processed sequentially, separated by a signal strength. HAP first decodes the weakest signal, and then decodes the next weakest signal. By repeating this procedure, it finally decodes the strongest signal. Experiments show that MISO, SDMA, and NOMA can improve the transmission rate of WD. The performance of MISObased hybrid SDMA and NOMA has been found to be superior to that of MISO-based SDMA-based data transfer systems, and the need for NOMA has also been identified. Eventually, it proved that multiple antennas and sector divisions are appropriate as a way to improve transmission rates, one of the important issues of WPCN.

The paper is organized as follows: Sect. 2 presents related work, and Sect. 3 describes our proposed Hybrid SDMA and NOMA approach, and the following Sect. 4, after experimenting with the Sum-Throughput and Fairness of the system proposed, presents the experimental results. In Sect. 5, we give our conclusions and future work.

\section{Related Works}

Incredible progress has been made in wireless technology in just over 40 years; the $5 \mathrm{G}$ and $6 \mathrm{G}$ will profoundly transform wireless telecommunications, paving the way for the network of future generations that will include more devices and allow faster communications and higher speeds. Because the RF signal convey energy and information; many research have been conducted in wireless powered communication networks (WPCN). Harvest-thentransmit protocol was proposed in [2], in which all users harvest the wireless energy broadcasted by the H-AP in the downlink (DL) and then send their own independent information to the HAP in the uplink (UL) via time-division multiple-access (TDMA). In [3], the researchers suggest user collaboration in the WPCN, in which the user who is closer to the $\mathrm{H}-\mathrm{AP}$ and has a stronger channel for DL energy harvesting as well as UL information sharing uses some of its allocated UL time and DL harvested energy to help transmit the information of the further user to the H-AP, resulting in a more balanced throughput. In [4], the researchers investigated a WPCN in which a single multi-antenna access point (AP) coordinates energy and information transmission to and from a group of single-antenna users. Therefore, when the AP has multiple antennas, the amount of energy transmitted to different users in the DL can be adjusted by designing different energy beamforming weights at the AP, while in the UL, all users can concurrently send data to the AP through SDMA. Since the access points (APs) operated by renewable energy are linked by a power line to allow simultaneous data and energy sharing, the HAP is called an energy source in the WPCN [5].

\subsection{MISO}

In mobile communication, a multi-antenna is a technology that processes signals using two or more antennas and improves transmission speed with a channel capacity proportional to the number of antennas without changing power and frequency. The technology is divided into spatial diversity, spatial multiplexing, and beamforming. Spatial 
diversity is a method that reduces fading effects and increases data reliability by transmitting one signal equally to multiple receiving antennas and selecting a good signal among receiving signals.

Spatial multiplexing is a method of simultaneously transmitting different data from the same resource (frequency, time, and code) to multiple receiving antennas, allowing simultaneous transmission through different path for each sector. Furthermore, because beamforming can adjust the signal strength according to the position angle of the receiving and sending nodes by adjusting the phase information for each antenna, thereby reducing interference with other nodes while minimizing signal attenuation.

Multiple antennas are divided into single-input-single-output (SISO), single- input multiple-output (SIMO), and multiple-input multiple-output (MISO) depending on the number of antennas on the transmitting and receiving nodes. SISO consists of a single antenna for both transmitting and receiving nodes with no effect of spatial diversity or spatial multiplexing. SIMO consists of a single antenna for the transmitting node and multiple antennas for the receiving node with no reception diversity effect or spatial multiplexing effect.

To increase minimum UL throughput, a technique to optimize energy allocation weight vector from HAP, CSI feedback phase length from WD, and UL BW based on MISO running in FDD mode was proposed [6]. In [7], a study was conducted to ensure the optimization and fairness of transmission rates by sending energy to all users with energy beamforming of HAP measured by the uplink channel according to the pilots sent by the user. Another technique for maximizing transmission rates by user node time allocation and beamforming according to MISO-based CSI was proposed in [8].

In MISO, the transmitting node has multiple antennas, and the receiving node has single antenna; there is no transmission diversity effect and spatial multiplexing effect. MIMO consists of multiple antennas for both transmitting and receiving nodes with both spatial diversity and spatial multiplexing effects. It is divided into single-userMISO and multi-user-MISO depending on the number of users. SU-MISO consists of one transmitting and receiving node. MU-MISO consists of multiple transmitting nodes and one receiving node. The MIMO systems have multiple number of antennas in transmitting and receiving end which can produce more data rates and capacity compared to SISO, SIMO, and MISO systems [9].

The proposed system is constructed based on MU-MISO as shown in Fig. 1. Multiple antennas can improve reliability and transmission speed due to spatial diversity or spatial multiplexing. However, WD uses a single antenna because multiple antenna

Fig. 1 Spatial multiplexing based on MU-MISO

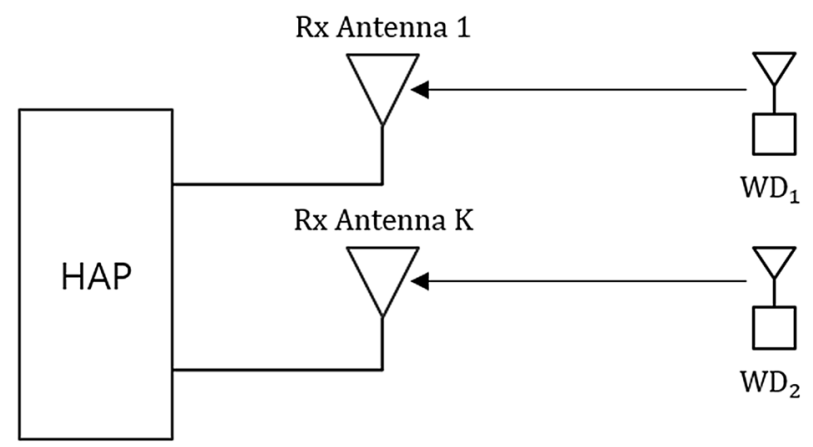


consumes more power than a single antenna and cannot guarantee the stability of WD. Our proposed system increases the transmission rate by applying sector division.

\subsection{SDMA}

Multiple Access (MA) is a technique for transmitting data while multiple nodes are jointly using a given resource (frequency, time, code). This technology enables multiple nodes to share resources and efficiently transfer data in resource-constrained mobile communications. The MA is divided into Orthogonal Multiple Access (OMA) and NOMA [10, 11]. In [12], the author conducted research on maximizing sum user energy efficiency while optimizing downlink beamforming vectors and uplink power and time allocation to users via TDMA and SDMA based on MISO. A technique was proposed in [13]; it demonstrated how to apply SDMA on a MISO basis and maximize the minimum transmission rate between users while optimizing DL and UL time allocation, DL energy beamforming, UL transmission power allocation, and receive beamforming together. Frequency Division Multiple Access (FDMA), Time Division Multiple Access (TDMA), and Code Division Multiple Access (CDMA) are orthogonal frequency division used as MA technology in 1G, 2G, and 3G respectively. Orthogonal Frequency Division Multiple Access (OFDMA) and Single-Carrier Frequency Division Multiple Access (SC-FDMA) have been adopted as an OMA technology standardized in 4G, Long-Term Evolution (LTE) and LTE-Advanced by 3rd Generation Partnership Project (3GPP).

The proposed system uses SDMA, which allows simultaneous transmission without interference in separated spaces while sharing frequency and time. This is because space multiplexing on multiple antennas in HAP allows WD to transmit signals through separate spaces without interference between sectors. Thus, it will induce a linear increase in channel capacity as much as the isolated space. Figure 2 shows a configuration diagram of a network in which multiple WDs for each sector are transmitting signal simultaneously to HAP using the same frequency, time, and code. Because simultaneous transmission is made by sector, it can be expected that the transmission rate will increase. However, beside

Fig. 2 Network configuration (SDMA)

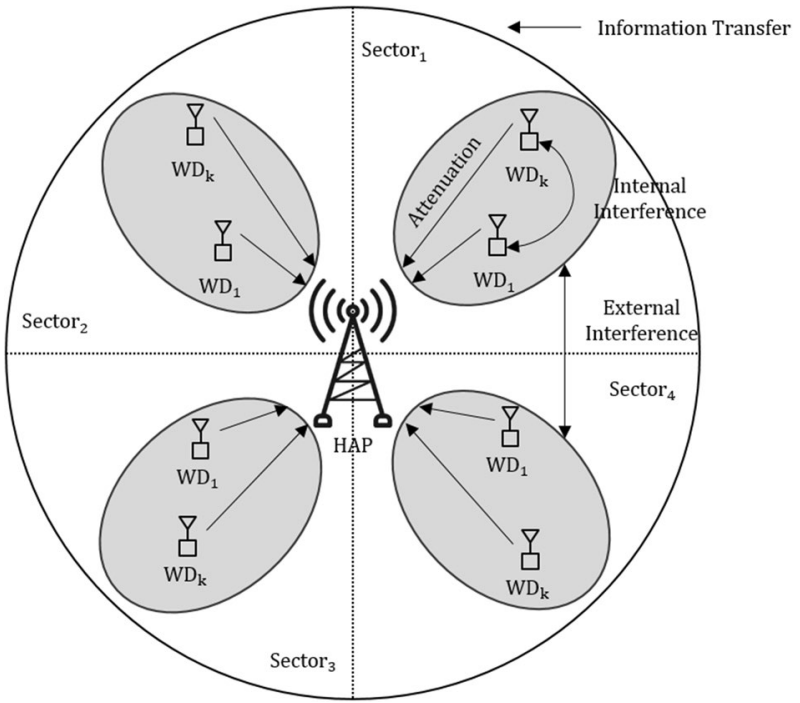


the interference of WDs in each sector and the interference inter-sector, signal attenuation can increase the signal loss rate of WDs.

\subsection{NOMA}

A method on how to allocate power in each cluster with a dynamic cooperative framework based on hybrid NOMA to ensure fairness among IoT devices and improve throughput was studied in [14]. In [11], the author has studied techniques for switching transmission modes for wireless channels and buffer states based on Hybrid NOMA and OMA. The author proposed in [15] a technique for allocating the optimal power for each user according to the minimum harvest energy required while operating as a group NOMA based on Hybrid TDMA and NOMA.

The proposed system uses NOMA, which allows multiple WDs to transmit simultaneously by multiplexing signals in the power domain while sharing frequency, time, code, and space. This is because multiple signals of WD are transmitted through Superposition Coding (SC), and the HAP performs Successive Interference Cancellation (SIC) [16]. SIC is a technology that processes multiple signals received from the receiver side simultaneously and processes the signal strength differences. In other words, the receiver decodes the received signal to extract the strong signal from the superimposed signal, and then removes the weak signal from the remaining signals [17]. As a result, interference between WD in the sector is removed and transmission rate is expected to be improved at the same time.

Because NOMA has no schedule, it can reduce transfer delay and overhead. Moreover, WDs with good channel conditions transmit signals with low power otherwise it transmits signals with large power and performs multiplexing in the power domain. This can improve fairness between multiple WDs. Through improved fairness, the transfer rates can be increased in poor wireless access environments. Thus, NOMA is considered a promising MA technology in the field of 5G mobile communications that require low latency, high throughput, high reliability, and large-scale connectivity [18]. To support the Internet of Things, 3GPP LTE Advanced was standardized under the name MUST (Multi-User Superposition Transmission) [19].

\section{Proposed Hybrid Protocol}

This section covers the proposed configuration of Hybrid SDMA and NOMA. We also describe the operating procedure for Hybrid and SDMA with flow charts and pseudo-codes according to the WD's distribution method.

\subsection{Hybrid SDMA and NOMA}

In the proposed system, one HAP equipped with multiple antennas, and many WDs equipped with a single antenna are randomly arranged in each sector as shown in Fig. 3. For a random WD's distribution, the distance and angle between WD and the HAP are randomly determined; however, for a fixed WD's distribution, the distance and angle between WD and the HAP are continuously increased. The parameters of the proposed system are described in Table 1.

The system model assumed in this paper is as follows. Each antenna in the HAP and each sector are located on the same line; the HAP and WD use oriented antennas. HAP 


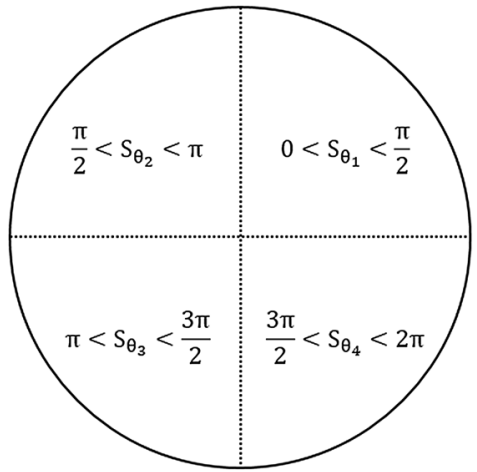

(a) Sector Division

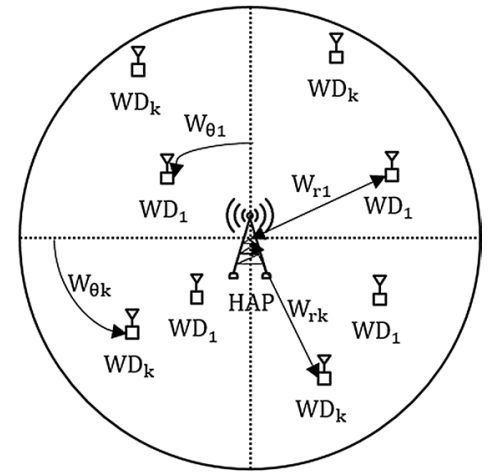

(b) Random WD Distribution

Fig. 3 WD distribution

Table 1 List of parameters

\begin{tabular}{ll}
\hline Parameter & Description \\
\hline $\mathrm{S}_{n}$ & Number of sector \\
$\mathrm{S}_{\theta}$ & Angle of each sector \\
$\mathrm{W}_{n}$ & Number of WD \\
$\mathrm{W}_{\mathrm{x},} \mathrm{W}_{y}$ & Coordinates of each WD \\
$\mathrm{W}_{\theta}$ & Angle of each WD \\
$D_{h}$ & Distance of each WD from HAP \\
$W_{s}$ & SNR of each WD \\
$S_{p}$ & Total power of one sector \\
$S_{t h}$ & Total transfer rate of one sector \\
$S_{t}$ & Total transfer rate for all sectors \\
$S_{S N R}$ & Total SNR of one sector \\
$S_{N} R_{w}$ & Total SINR of one sector \\
$S I N R_{t h}$ & Difference of Threshold signal strength \\
$N_{p}$ & Noise power of each WD \\
$H_{g}$ & Channel gain for each WD \\
$\mathrm{W}_{p}$ & Power of each WD \\
$I_{p}$ & Maximum power in one sector \\
\hline
\end{tabular}

also broadcasts power to WD within all Sector. With Channel Reciprocity, the channel gain for UL and DL between HAP and WD is the same, and the synchronization between HAP and all WD is correct. In this state, all WDs transmit signals to the HAP, consuming all the power received from the HAP without loss. The channel uses the Quasi-static flat-fading model; therefore, Channel Gain is constant for each frame, and HAP fully knows the Channel State Information (CSI).

Figure 4 shows the configuration diagram of the proposed system. HAP with multiple antennas receives signals from multiple WDs. WDs for each sector transmits signals to the HAP with different signal strength, and the HAP decodes those signals according to the SIC. 
Fig. 4 System model configuration (hybrid SDMA and NOMA)

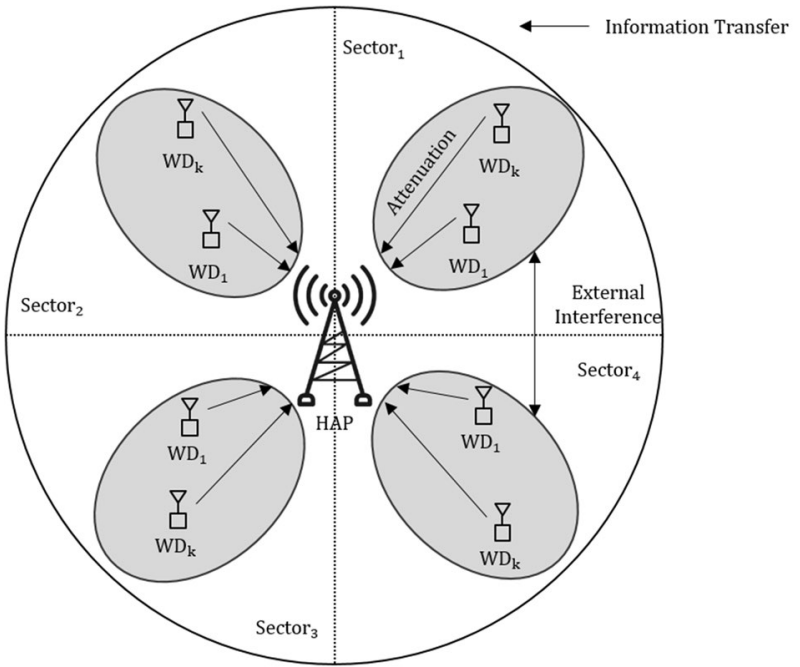

The signal strength of each WD must be exponentially different from each other in order to be decoded [20]. Signal loss rates of WD can occur due to interference between sectors and signal attenuation, but an increased transmission rate can be expected because NOMA reduces interference between WDs in each sector. In each sector, multiple WD transmits a data to the HAP with weak signals if the WD is close to the HAP meanwhile the far WD transmits a data to the HAP with strong signals. The HAP can only receive signals from WDs with relative differences in strength. The received signal is used to calculate the transmission rate for each sector, and Sum-Throughput is calculated by summing them. Therefore, according to Jain's Fairness, fairness is measured by the transmission rate per sector.

As shown in Fig. 5, WD, which differs in distance from HAP, simultaneously transmits signals to one HAP. Upon receiving the superimposed transmitted signals, the HAP uses the SIC to decode the signals from both WDs.

The HAP decodes the weak signal, and then, the strong signal. As a result, the channel capacity of WD1 is as follows:

$$
R_{1}=\log _{2}\left(1+\frac{P_{1}\left|h_{1}\right|^{2}}{N_{1}}\right)
$$
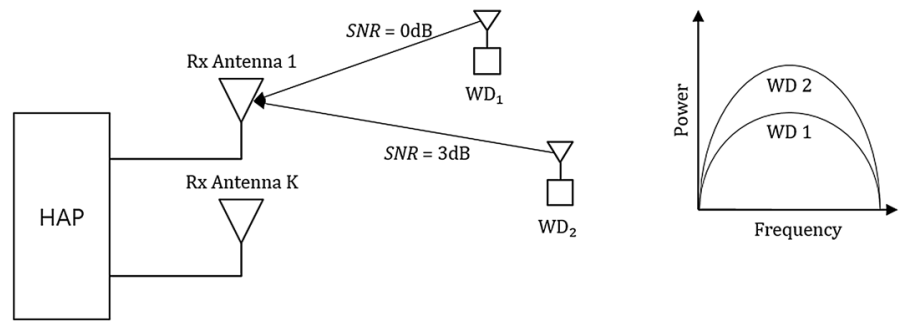

Fig. 5 Multiplexing based on NOMA 
The channel gain is h, the power level of each WD is $\mathrm{P}$, and the noise per WD is $\mathrm{N}$, and the channel capacity of WD2 is as follows:

$$
R_{2}=\log _{2}\left(1+\frac{P_{2}\left|h_{2}\right|^{2}}{P_{1}\left|h_{2}\right|^{2}+N_{2}}\right)
$$

\subsection{Flow Chart}

Figure 6 shows the operating procedure of Hybrid SDMA and NOMA with a random distribution of the WD. First, we set the signal strength threshold, the number of sectors, and the number of WDs. A cell is divided into equal sizes by the number of sectors, and depending on the threshold, the amount of WD signals that can be received varies. Moreover, the coordinates and angles of each WD are set randomly, and the distance difference from the HAP is determined by the value set.

The Signal Noise Ratio (SNR) of each WD is constructed differently, proportional to the power strength and channel gain, and inversely proportional to the noise power and distance difference from the HAP. The HAP receives only SNR above the threshold of the signal strength difference among the overlapped signals of WDs by Sector. The sum of the SNR received is inversely proportional to the interference power of the other sector.

In addition, according to the channel capacity formula, the transmission rate of each sector is calculated, and Sum-Throughput is obtained by sum of the transmission rates of each sector. Finally, fairness is calculated with the transmission rate by Sector. The operating procedure for SDMA with the WD's distribution method fixed is shown in Fig. 7. Most of the operating procedures are shown in Fig. 6, and other parts are shown below. SDMA
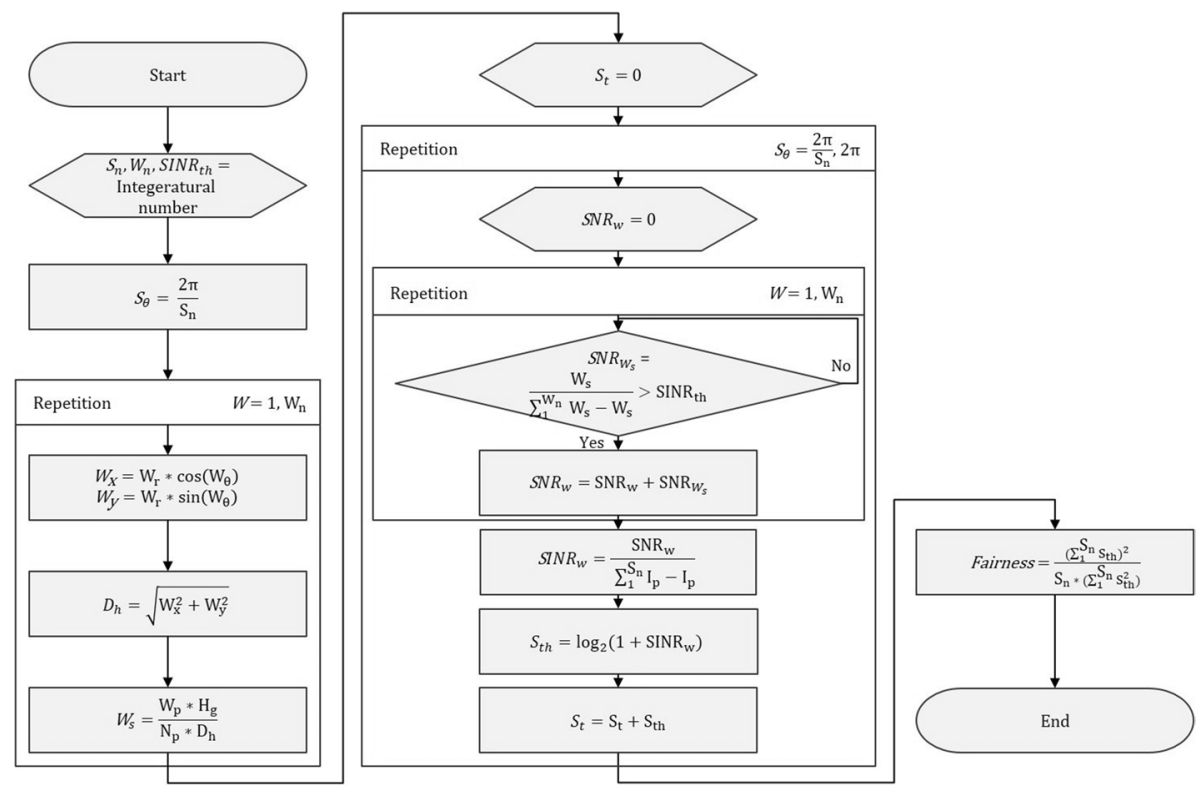

Fig. 6 Flowchart (hybrid SDMA and NOMA-random) 

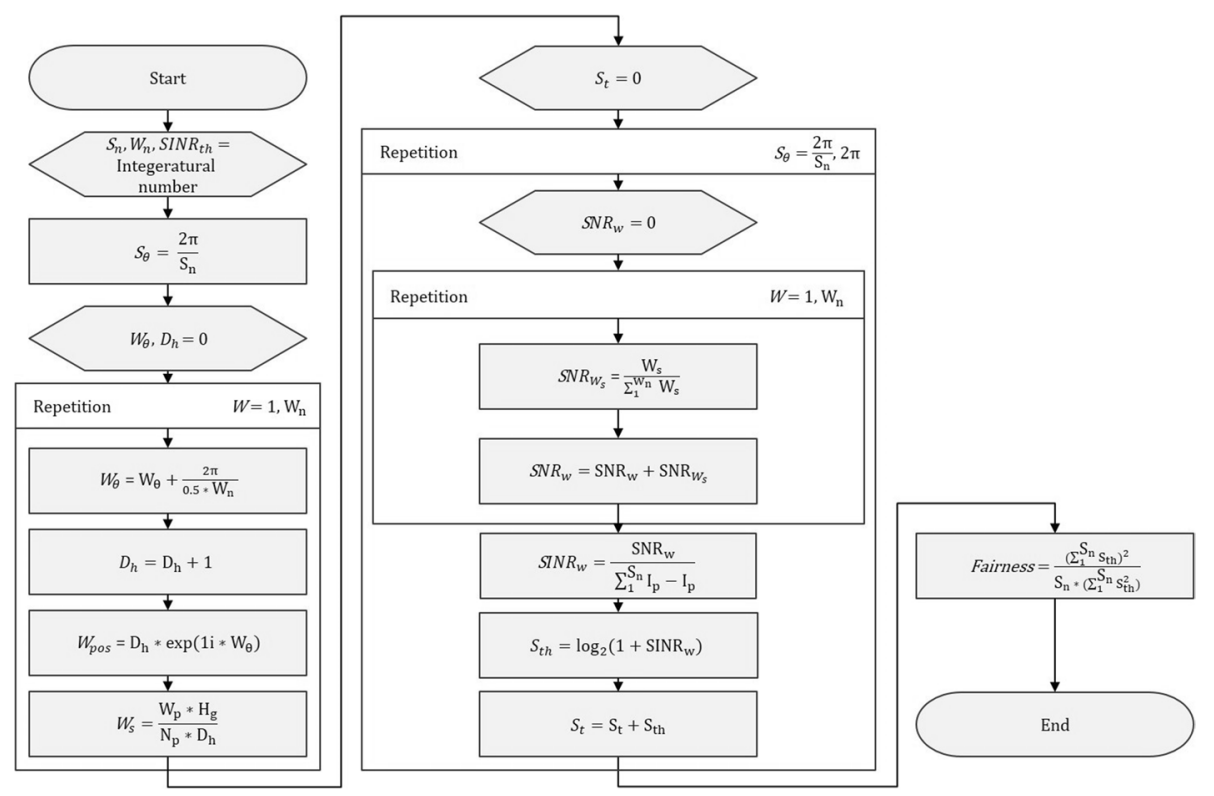

Fig. 7 Flowchart (SDMA-Fixed)

does not set this because it does not require a threshold for the signal strength difference. Because it is a fixed distribution, the coordinates and angles of each WD are increased consistently.

Sum-Throughput is calculated as the sum of SNR for each WD inversely proportional to the sum of SNR for multiple WDs by Sector.

\subsection{Pseudo-Code}

The operating procedures for Hybrid SDMA and NOMA with the WD's fixed distribution method are shown in the Algorithm 1. 
Input: NumberOfSector, NumberOfNode, SINR

Output: SumOfThroughputBySector, Fairness

1 sector $\longleftarrow 1$

2 node $\longleftarrow 1$

$3 \quad \theta \longleftarrow 0$

4 distance $\longleftarrow 0$

5 SNR $\longleftarrow 0$

6 SumThroughputBySector $\longleftarrow 0$

7 TotalThroughput $\longleftarrow 0$

8 AngleBySector $\longleftarrow 2 \pi /$ NumberOfSector

9 while node < NumberOfNode do

$10 \mid \theta \longleftarrow \theta+(2 \pi /(0.5 *$ NumberOfNode $))$

11 distance $\longleftarrow$ distance +1

$12 \quad S N R \longleftarrow($ PowerOf Node $*$ ChannelGain $) /($ NoisePower $*$ distance $)$

13 while node $<$ NumberOfNodes do

$14 \quad$ Sort NodeBySector

15 end

16 while sector $<$ NumberOfSector do

17

18

19

20

21

22

23

24

25

26

I SNROfSector $\longleftarrow \sum S N R O f N o d e B y S e c t o r$

end

while sector $<$ NumberOfSector do

| MaxPower BySector $\longleftarrow \max ($ PowerOf NodeBySector)

end

while sector < NumberOfSector do

| SumMaxPower BySector $\longleftarrow \sum$ MaxPower BySector

end

while sector < NumberOfSector do

InterferencePower BySector $\longleftarrow$

$\sum$ MaxPower BySector - MaxPower BySector

end

while sector $<$ NumberOfSector do

28

29

30

31

32

33

34

35

36

37

38

39

40

41

if $(S N R /(S N R O f S e c t o r-S N R))>$ ThresholdValueOfSINR then

| $S N R \longleftarrow S N R /(S N R O f S e c t o r-S N R)$

end

$S I N R \longleftarrow S N R /$ InterferOfPower

Throughput $\longleftarrow \log 2(1+S I N R)$

SumOfThroughputBySector $\longleftarrow \sum$ ThroughputBySector + Throughput end

while sector $<$ NumberOfSector do

| TotalThroughput $\longleftarrow \sum S u m O f T h r o u g h p u t B y$ Sector

end

while sector $<$ NumberOfSector do

Fairness $\longleftarrow$
(TotalThroughp

(TotalThroughput $\left.{ }^{2}\right) /($ NumberOfSector $*$ SumOfThroughput $)$

42 end

Algorithm 1: Hybrid SDMA and NOMA-Fixed

The operating procedure for SDMA with the WD's random distribution method is shown in Algorithm 2. 
Algorithm 2: SDMA-Random

Input: NumberOfSector, NumberOfNode, SINR

Output: SumOfThroughputBySector, Fairness

1 sector $\longleftarrow 1$

2 node $\longleftarrow 1$

$30 \longleftarrow 0$

$4 r \longleftarrow 0$

$5 \quad x \longleftarrow 0$

$6 y \longleftarrow 0$

7 distance $\longleftarrow 0$

$8 S N R \longleftarrow 0$

9 AngleBySector $\longleftarrow 2 \pi / N u m b e r O f$ Sector

10 while node < NumberOfNode do

$11 \mid \theta \longleftarrow \operatorname{rand}(\theta)$

$12 \quad r \longleftarrow \operatorname{rand}(r)$

$13 \quad x \longleftarrow r \cos \theta$

$14 \quad y \longleftarrow r \sin \theta$

$15 \quad$ distance $\longleftarrow \sqrt{\left(x^{2}\right)+\left(y^{2}\right)}$

$16 \quad S N R \longleftarrow($ PowerOfNode $*$ ChannelGain $) /($ NoisePower $*$ distance $)$

17 end

18 while node < NumberOfNode do

19 SortNodeBySector

20 end

21 while sector $<$ NumberOfSector do

22 SNRofSector $\longleftarrow \sum S N$ Rof NodeBySector

23 end

24 while sector $<$ NumberOfSector do

25 | MaxPower BySector $\longleftarrow \max ($ Power Of NodeBySector)

27 while sector $<$ NumberOfSector do

28 SumMaxPower BySector $\longleftarrow \sum$ MaxPowerBySector

29 end

30 while sector $<$ NumberOfSector do

$31 \quad$ Inter ference Power BySector $\longleftarrow$

$\sum$ MaxPower BySector - MaxPower BySector

32 end

33 while sector < NumberOfSector do

$34 \quad S N R \longleftarrow S N R / S N R$ Rof Sector

$35 \quad S I N R \longleftarrow S N R /$ Inter ferencePower

$36 \quad$ Throughput $\longleftarrow \log 2(1+S I N R)$

37 SumOfThroughputBySector $\longleftarrow \sum$ ThroughputBySector + Throughput

38 end

39 while sector < NumberOfSector do

40 | TotalThroughput $\longleftarrow \sum$ SumOfThroughputBySector

41 end

42 while sector $<$ NumberOfSector do

43

Fairness $\longleftarrow$

44 end

(TotalThroughput $\left.{ }^{2}\right) /($ NumberOfSector $*$ SumOfThroughputBySector $)$ 
Table 2 Simulation parameter's values

\begin{tabular}{ll}
\hline Notation & Value \\
\hline$S n$ & Variable \\
$W_{n}$ & 100 \\
$S N R_{t h}$ & Variable \\
$N_{p}$ & $-60 \mathrm{dBm}$ \\
$H_{g}$ & $10^{-3 * \mathrm{D}_{\mathrm{h}}^{-2}}$ \\
$W_{p}$ & $30 \mathrm{dBm}$ \\
\hline
\end{tabular}

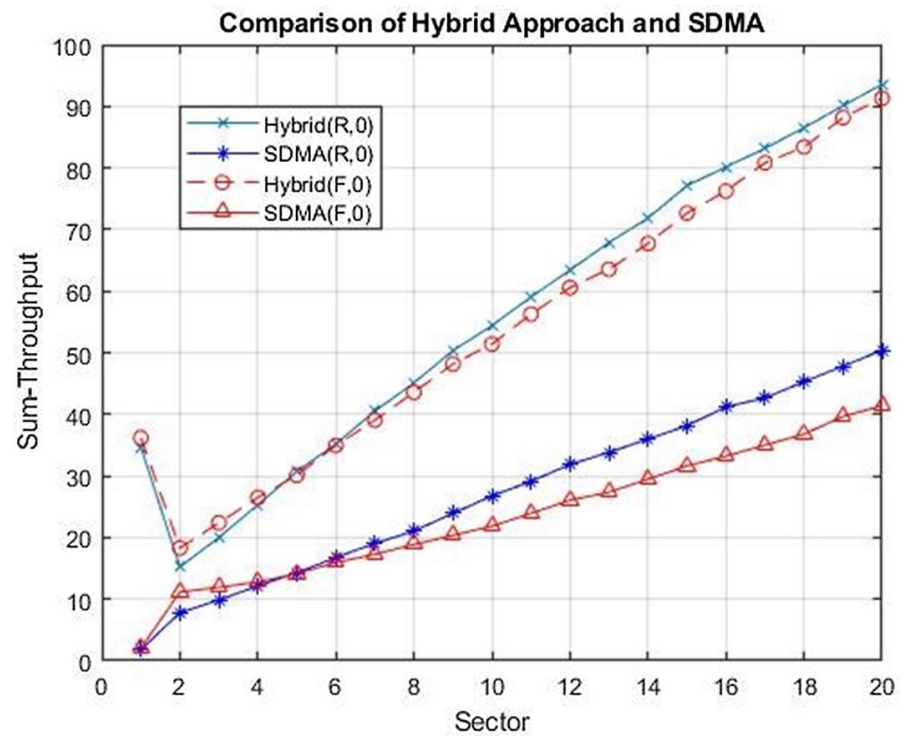

Fig. 8 Comparison of sum-throughput as function of sectors

\section{Performance Evaluation}

The experiment was implemented with MATLAB R2020a to calculate Sum-Throughput and Fairness. The total throughput is the sum of the channel capacity by sector and fairness indicates how fair the throughput by sector is with the channel capacity by sector. Jain's Fairness becomes 1 if the throughput of each sector is the same, and as the difference increases, it approaches 0 . The experimental result is the average of the results obtained after performing 1000 times.

In experimental results, according to the parameter's values as described in Table 2, the Hybrid approach is the proposed system, and SDMA is the comparison system. $\mathrm{R}$ is the case for random distribution of WD, while $\mathrm{F}$ is the case for fixed distribution, and $0,3,6$, and 10 are the thresholds for the signal strength difference.

Figure 8 shows the comparison of Sum-Throughput by a number of sectors for the Hybrid approach and SDMA. Hybrid can be found to perform better in all sectors than SDMA. In addition, both the Hybrid approach and SDMA have a random distribution 
of WDs from more than a specific number of sectors, better performance than fixed distribution.

The comparison of sector-by-sector fairness for Hybrid and SDMA is shown in Fig. 9. Hybrid can be seen to perform better when the number of sectors is equal to 4 than SDMA. Moreover, Hybrid performs better with a random distribution of WD up to sector 18 than with fixed distribution, and SDMA performs better with a random distribution of WD across all sectors.

As the number of sectors increases, the number of WDs located within each sector decreases. As a result, calculating the channel capacity as the sum of SNR for each sector increases the transmission rate. Therefore, Sum-Throughput increases as the number of sectors increases; the necessity of NOMA that guarantees fairness can be confirmed as a result of the experiment that the Hybrid Approach is superior to SDMA.

Figure 10 shows the comparison of the number of sectors, trade-off considering SumThroughput and Fairness together for Hybrid, depending on the distribution method of WD. Both Hybrid and SDMA show that the number of sectors that are trade-off is 17, and the performance on sector 17 is slightly better when WD is placed randomly than when WD is placed fixedly.

Figure 11 shows the comparison of the Sum-Throughput for each threshold of the difference in the number of sectors and signal strength for the Hybrid Approach and SDMA. We can confirm that Hybrid outperforms SDMA in all thresholds and number of sectors and that smaller thresholds improve performance. Hybrid performs better at each threshold when WD is randomly distributed from a certain number of sectors than fixed distribution.

The results of comparing fairness by the threshold of the number of sectors and signal strength differences for Hybrid and SDMA are shown in Fig. 12. Hybrid can confirm that if the threshold is smaller, the performance is better, and hybrid performs better at each threshold when WD is randomly distributed than fixedly. The higher the number of signals that the HAP will receive, the lower the threshold for the difference in signal power.

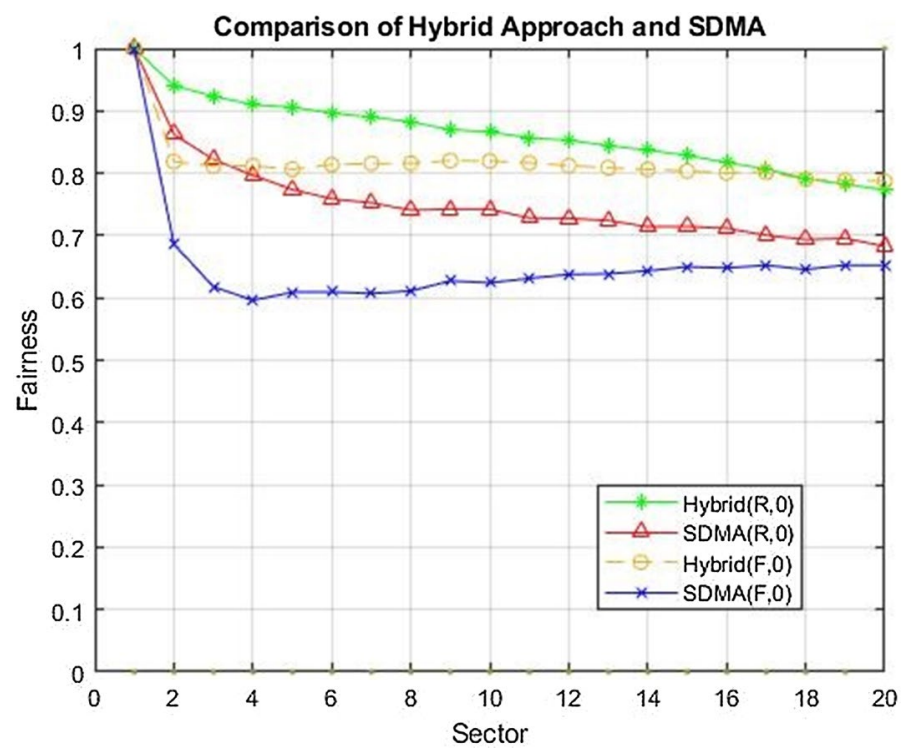

Fig. 9 Comparison of fairness according to the number of sector 


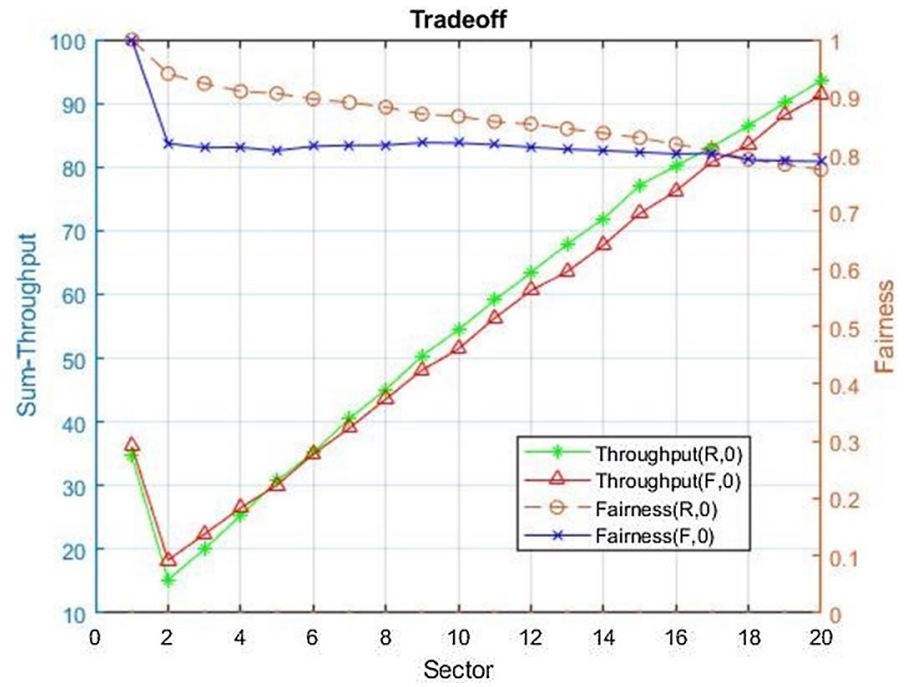

Fig. 10 Tradeoff between sum-throughput and fairness for hybrid approach

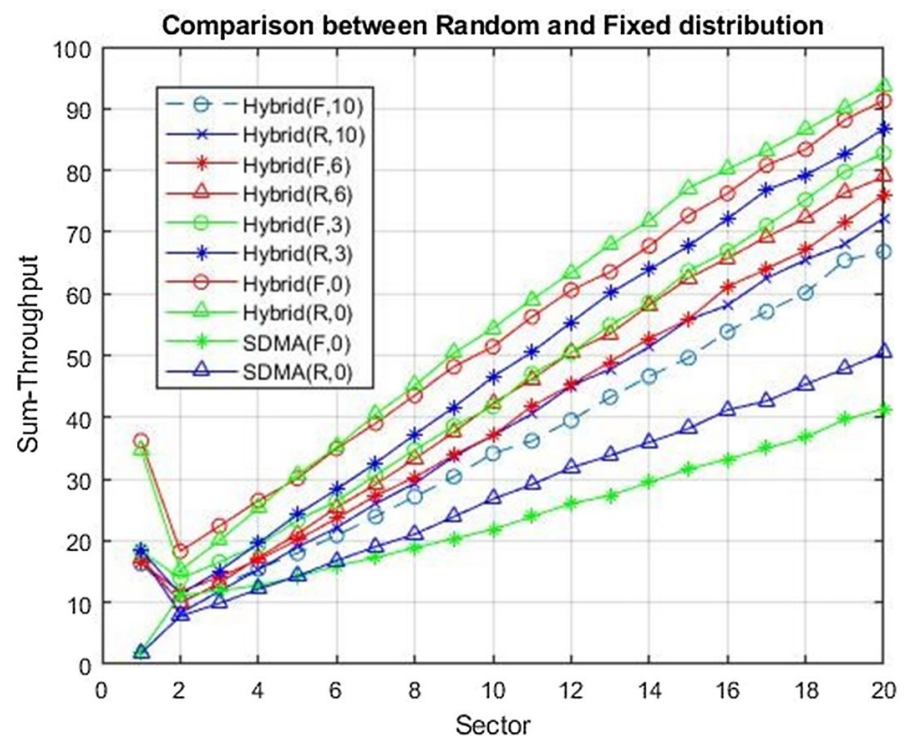

Fig. 11 Sum-throughput comparison between random and fixed distribution of WDs

Increasing the SNR total for each sector will also increase the transmission rate. As a result, smaller thresholds work better.

Lifetime in the wireless sensor network depends on battery capacity, and wireless power transfer (WPT) has been proposed in the past to improve lifetime of sensor nodes [21]. This WPT will provide periodic power to the nodes and also improve the lifetime 


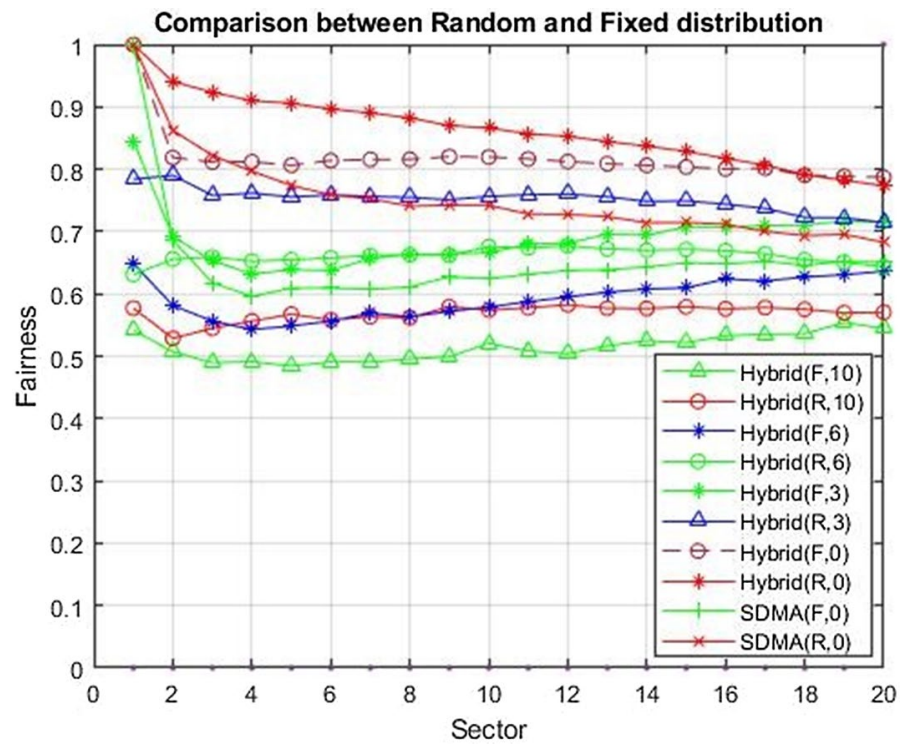

Fig. 12 Fairness Comparison between random and fixed distribution of WDs

of the sensor node. The formula for calculating end-to-end power transfer efficiency over WPT is as follows:

$$
e=\frac{P_{d c}^{r}}{P_{d c}^{t}}=\frac{P_{r f}^{t}}{P_{d c}^{t}} \frac{P_{r f}^{r}}{P_{r f}^{t}} \frac{P_{d c}^{r}}{P_{r f}^{r}}
$$

As shown in Fig. 13, $P_{d c}^{t}$ is input DC power and $P_{r f}^{t}$ is output RF power; $P_{r f}^{r}$ is input RF power and $P_{d c}^{r}$ is output DC power. $P_{r f}^{t} / P_{d c}^{t}$ represents DC-to-RF conversion efficiency; $P_{r f}^{r} / P_{r f}^{t}$ represents RF-to-RF transmission efficiency, and $P_{d c}^{r} / P_{r f}^{r}$ represents RF-to-DC conversion efficiency.

In terms of wireless sensor network [22, 23], maximizing $P_{r f}^{r} / P_{r f}^{t}$ for end-to-end power transfer efficiency over WPT will improve the Sum-Throughput and the lifetime through maximization of $P_{d c}^{r}$. The transmitting power from HAP can be seen as one of several ways to increase $P_{r f}^{r} / P_{r f}^{t}$.

Signal attenuation can cause those nodes to lose energy if the transmission power is small, depending on the distance between the HAP and the node. When the battery of node discharge, the transfer fails as well, resulting in a lower Sum-Throughput and a shorter lifetime. Therefore, improving end-to-end power transmission efficiency by optimizing $P_{r f}^{r} / P_{r f}^{t}$ can maximize the transmission power broadcasting from HAP to all nodes in the cell. As a result, the Sum-Throughput and the lifetime will both grow over time. On the other hand, strong

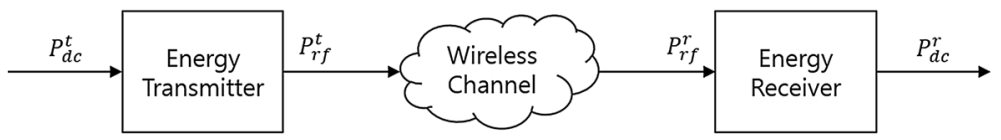

Fig. 13 The block diagram of generic WPT system [22] 
power will cause issues like increasing interference, which reduces the throughput of neighbor nodes, thereby demanding optimal power allocation for node positioning states.

\section{Conclusion}

To improve performance in WPCN, we propose MU-MISO-based Hybrid SDMA and NOMA. Spatial multiplexing with multiple antennas and SDMA alone will improve SumThroughput. However, as the number of WDs in the same sector increases, the interference may increase. As a result, you cannot expect as much throughput as the number of WDs in the sector. Therefore, we proposed a method for multiplexing the signal of the same sector of multiple WDs with NOMA in the power domain to simultaneously output the signal. As the proposed method, the fact that Sum-Throughput and Fairness are superior to that of the existing SDMA and the number of sectors that are tradeoff were confirmed by the experimental results. In the end, it proved that SDMA can be improved. However, batterypowered WDs are not sufficiently recharged and cannot transmit signals continuously. In the research, it is necessary to study a method that can guarantee the continuous signal transmission of WD.

Funding This work was supported by the National Research Foundation of Korea (NRF) grant funded by the Korea Government (MSIT) (No. NRF-2019R1A2C1009894).

Availability of Data and Material The data that support the findings of this study are available on request from the corresponding author, I. J. The data are not publicly available due to their containing information that could compromise the privacy of research participants.

Code Availability The data for this project are confidential, but may be obtained with Data Use Agreements with the Computer Software Department of Hanyang University. Researchers interested in access to the data may contact Juhyun Maeng at jhmaeng @ hanyang.ac.kr, also see http://wm.hanyang.ac.kr/xe/contact. It can take some months to negotiate data use agreements and gain access to the data. The author will assist with any reasonable replication attempts for two years following publication.

\section{Declarations}

Conflict of interest The authors declare that they have no conflict of interest.

Open Access This article is licensed under a Creative Commons Attribution 4.0 International License, which permits use, sharing, adaptation, distribution and reproduction in any medium or format, as long as you give appropriate credit to the original author(s) and the source, provide a link to the Creative Commons licence, and indicate if changes were made. The images or other third party material in this article are included in the article's Creative Commons licence, unless indicated otherwise in a credit line to the material. If material is not included in the article's Creative Commons licence and your intended use is not permitted by statutory regulation or exceeds the permitted use, you will need to obtain permission directly from the copyright holder. To view a copy of this licence, visit http://creativecommons.org/licenses/by/4.0/.

\section{References}

1. Bi, S., \& Ho, C. K. (2015). Wireless powered communication: Opportunities and challenges. IEEE Communications Magazine, 53(4), 117-125. 
2. Ju, H., \& Zhang, R. (2014). Throughput maximization in wireless powered communication networks. IEEE Transactions on Wireless Communications, 13, 418-428.

3. Ju, H., \& Zhang, R. User cooperation in wireless powered communication networks. In 2014 IEEE Global Communications Conference (pp. 1430-1435). https://doi.org/10.1109/GLOCOM.2014.70370 09.

4. Liu, R.Z.L., \& Chua, K.-C. (2014). Multi-antenna wireless powered communication with energy beamforming. Transactions onCommunications, 13, 4349-4361.

5. Zheng, E. J. G., Ho, Z., \& Ottersten, B. (2014). Information and energy cooperation in cognitive radio networks. IEEE Transactions on Signal Processing, 62, 2290-2303.

6. Ahmadian, A. (2018) Throughput optimization in FDD MU-MISO wireless powered communication networks. CoRR, vol. abs/1809.10830.

7. Zhang, R., Guan, Y. L., Yang, G., \& Ho, C. K. (2015). Throughput optimization for massive MIMO systems powered by wireless energy transfer. IEEE Journal on Selected Areas in Communications, 33(8), 1640-1650.

8. Shen, C., Li, X., Zhong, Z., Sun, Q., \& Zhu, G. (2014). Joint beamforming design and time allocation for wireless powered communication networks. IEEE Communications Letters, 18(10), 1783-1786.

9. Ashish Kumar Sarangi, A.D. (2018) Capacity comparison of SISO, SIMO, MISO MIMO systems. In 2018 ICCMC, pp. 798-801.

10. Ding, Z., Wang, Z., Chen, S., Hanzo, L., Dai, L., Wang, B. (2017). A survey of non-orthogonal multiple access for 5g. In 2017 IEEE 86th Vehicular Technology Conference (VTCFall), Toronto, ON, Canada.

11. Xu, P., Quan, J., Yang, Z., Chen, G., \& Ding, Z. (2019). Performance analysis of buffer-aided hybrid NOMA/OMA in cooperative uplink system. IEEE Access, 7, 11.

12. Hunwoo Lim T.H. (2019). Energy-efficient miso wireless powered communications. In 2019 16th IEEE Annual Consumer Communications Networking Conference (CCNC).

13. Chua, K.-C., Liu, L., \& Zhang, R. (2014). Multi-antenna wireless powered communication with energy beamforming. IEEE Transactions on Communications, 62(12), 4349-4361.

14. Shao, X., Yang, C., Chen, D., Zhao, N., \& Yu, F. (2018). Dynamic IOT device clustering and energy management with hybrid NOMA systems. IEEE Transactions on Industrial Informatics, 14, 4622-4630.

15. Al-Obiedollah, H., et al. (2019) On energy harvesting of hybrid TDMA-NOMA systems. In 2019 IEEE Global Communications Conference (GLOBECOM), 2019 (pp. 1-6). https://doi.org/10.1109/ GLOBECOM38437.2019.9013805.

16. Kishiyama, Y., Li, A., Harada, A., Nakamura, T., Benjebbour, A., \& Saito, Y. (2013). Concept and practical considerations of non-orthogonal multiple access (NOMA) for future radio access. In Intelligent Signal Processing and Communications Systems (ISPACS), pp. 770-774.

17. Sen, S., Santhapuri, N., Choudhury, R.R., \& Nelakuditi, S. (2010) Successive interference cancellation: a back-of-the-envelope perspective. In Proceedings of the 10th ACM Workshop on Hot Topics in Networks, (Monterey, CA, USA), pp. 1-6.

18. Dai, L., Wang, B., Yuan, Y., Han, S., Chih-Lin, I., \& Wang, Z. (2015). Non-orthogonal multiple access for $5 \mathrm{~g}$ : Solutions, challenges, opportunities, and future research trends. IEEE Communications Magazine, 53(9), 74-81.

19. 3GPP (2017). 3GPP TDOC rp-150496: Study on downlink multiuser superposition transmission for LTE. 2015. Accessed 16 Sep 2017.

20. Gitlin, R., Al Rabee, F., \& Davaslioglu, K. (2017) The optimum received power levels of uplink nonorthogonal multiple access (NOMA) signals. In 2017 IEEE 18th Wireless and Microwave Technology Conference (WAMICON).

21. Xie, L., Shi, Y., Hou, Y., \& Lou, A. (2013). Wireless power transfer and applications to sensor networks. Wireless Communications IEEE, 20, 140-145.

22. Zeng, Y., Clerckx, B., \& Zhang, R. (2016). Communications and signals design for wireless power transmission. IEEE Transactions on Communications, 65, 2264-2290.

23. Clerckx, B., Zhang, R., Schober, R., Ng, D. W. K., Kim, D. I., \& Poor, H. V. (2018). Fundamentals of wireless information and power transfer: From RF energy harvester models to signal and system designs. IEEE Journal on Selected Areas in Communications, 37, 4-33.

Publisher's Note Springer Nature remains neutral with regard to jurisdictional claims in published maps and institutional affiliations. 

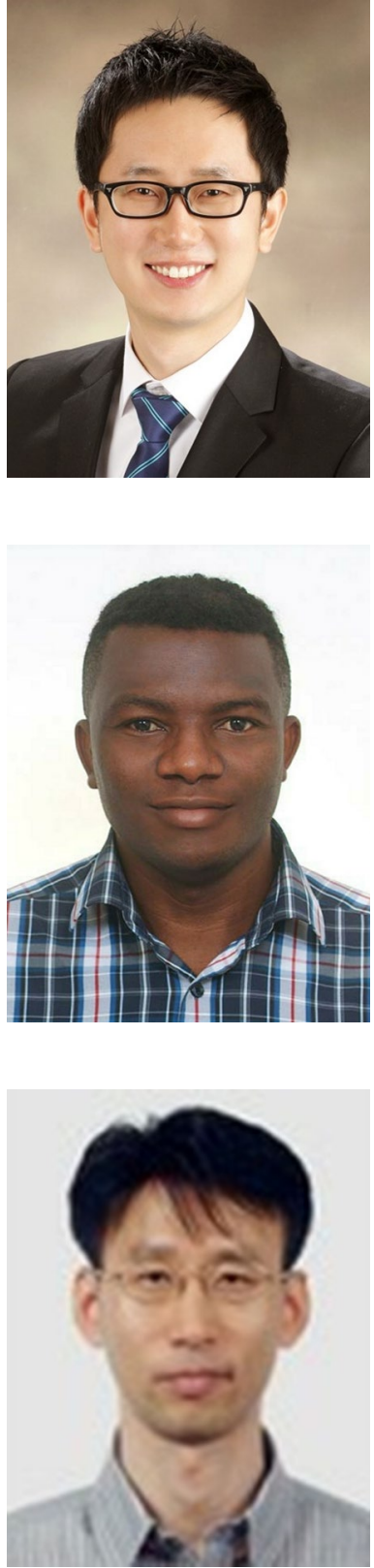

Juhyun Maeng received the BS in information communications engineering from Sejong University, Seoul, Korea in 2011, and the MS in electronic computer and communications engineering from Hanyang University, Seoul, Korea in 2013. From 2013 to 2017, he had been a researcher with the Center of Human-centered Interaction for Coexistence. He is currently pursuing the Ph.D. in software engineering at Hanyang University, Seoul, Korea. His research interests include wireless sensor networks, wireless powered communication networks, blockchain, and non-terrestrial network.

Mwamba Kasongo Dahouda received the BS in information system engineering from University Protestant of Lubumbashi (UPL), Lubumbashi, DR Congo in 2016, he had been a teaching assistant for one year at UPL, and the MS in software engineering from Hanyang University, Seoul, Korea in 2020. He is currently pursuing the Ph.D. in software engineering at Hanyang University, Seoul, Korea. His research interests include artificial intelligence, wireless powered communication networks, and non-terrestrial network.

Inwhee Joe received his BS and MS in electronics engineering from Hanyang University, Seoul, Korea, and his $\mathrm{Ph} . \mathrm{D}$. in electrical and computer engineering from Georgia Institute of Technology, Atlanta, GA in 1998. Since 2002, he has been a faculty member in the Division of Computer Science and Engineering at Hanyang University, Seoul, Korea. His current research interests include mobile internet, cellular system, and PCS, wireless sensor networks, mobile ad-hoc networks, multimedia networking, and performance evaluation. 